\title{
PERCOLACIÓN Y LIXIVIACIÓN DE NUTRIMENTOS EN SUELOS BANANEROS AL ESTE DEL RÍO REVENTAZÓN, COSTA RICA ${ }^{1}$
}

\author{
Jorge Acón-Ho ${ }^{2}$, Luis Alpízar-Oses ${ }^{3}$, Rodolfo WingChing-Jones ${ }^{4}$
}

\begin{abstract}
RESUMEN
Percolación y lixiviación de nutrimentos en suelos bananeros al este del río Reventazón, Costa Rica. El objetivo de este trabajo fue determinar la pérdida por lixiviación de $\mathrm{Ca}, \mathrm{Mg}, \mathrm{K}$ y nitrógeno nítrico en suelos bananeros. Entre el 2003-2004 en la Finca Venecia, situada en Matina de Limón, Costa Rica, se emplearon tres fuentes de fertilizante nitrogenadas: urea (U), nitrato de amonio (NA) y sulfato de amonio (SA). Se muestreó a una profundidad de $60 \mathrm{~cm}$ utilizando lisímetros, y el volumen del agua percolada (D) se calculó mediante el método indirecto del balance hídrico del suelo (BHs). En pH, independientemente de las fechas de muestreo, no se determinaron diferencias estadísticas. La concentración de calcio (Ca) en los lixiviados para los diferentes tratamientos durante las diferentes épocas de muestreos no fue significativa, pero se presentaron diferencias entre el tratamiento con urea con respecto a los tratamientos de NA y el SA, sin embargo, no se encontraron diferencias entre el NA y SA; tampoco entre los promedios de las concentraciones de magnesio $(\mathrm{Mg})$ y el potasio $(\mathrm{K})$ en los lixiviados. Para el nitrógeno nítrico, sólo hubo diferencias significativas entre el NA respecto a la $\mathrm{U}$ y al SA, tanto en forma general, como entre fechas de muestreo.
\end{abstract}

Palabras clave: fuentes de nitrógeno, pérdida de cationes, fluctuación de nutrimentos en el suelo, nitrógeno nítrico en el suelo.

\begin{abstract}
Percolation and leaching of nutrients in banana soils to the East of the Reventazón river, Limón, Costa Rica. The objective of this study was to detwermine leaching losses of $\mathrm{Ca}, \mathrm{Mg}, \mathrm{K}$ and nitric $\mathrm{N}$ in banana soils. The work was conducted between 2003-2004 in the Venecia Farm in Matina, Limón, Costa Rica. Three nitrogen fertilizer sources were used: urea $(\mathrm{U})$, ammonium nitrate $(\mathrm{aN})$ and ammonium sulfate (HS). Sampling was conducted at a depth of $60 \mathrm{~cm}$ using lysimeters and the volume of percolated water (D) was calculated using the indirect method of soil water balance (BHs). Irrespective of the sampling dates no statistical differences were determined found for $\mathrm{pH}$. The concentration of calcium $(\mathrm{Ca})$ in the leachate for different treatments during different times of sampling, was not significant, but significant differences were found between treatment with $U$ with respect to treatment of NA and SA. No differences between the NA and SA or in the average concentrations of magnesium $(\mathrm{Mg})$ and potassium $(\mathrm{K})$ in the leachate were observed. In the case of nitric nitrogen, significant differences were detected only between the NA regarding the U and SA, both in general and between sampling dates.
\end{abstract}

Keywords: nitrogen sources, cation loss, soil nutrients fluctuation, soil nitric nitrogen.

\footnotetext{
1 Recibido: 31 de julio, 2012. Aceptado: 28 de octubre, 2013. Trabajo de Graduación del primer autor. Programa de Estudios de Posgrado en Ciencias Agrícolas y Recursos Naturales. Universidad de Costa Rica.

2 San José, Costa Rica. jorge.acon@ice.co.cr

3 Departamento de Investigación e Innovación, Instituto de Innovación y Transferencia de Tecnología Agropecuaria (INTA)/Ministerio de Agricultura y Ganadería (MAG). San José, Costa Rica. lalpizaroses@yahoo.es

4 Escuela de Zootecnia, Centro Investigación en Nutrición Animal. Universidad de Costa Rica. San José Costa Rica. rodolfo.wingching@ucr. ac.cr 


\section{INTRODUCCIÓN}

El banano es una planta de rápido crecimiento y de alto rendimiento, por lo que es sensible a carencias o excesos de nutrimentos (López 1991). Un adecuado manejo nutricional implica conocer las diferentes vías de aportes y pérdidas de los nutrimentos en el agroecosistema bananero (López y Solís 1992), para así poder suministrar estos nutrimentos perdidos de forma in situ o a través de la aplicación de fertilizantes sintéticos, enmiendas y de materia orgánica.

Del total de nutrimentos inmovilizados por la planta de banano, una parte regresa al suelo mediante los residuos de cosecha (Vargas y Flores 1995), mientras que deben reponerse los removidos por el fruto durante la cosecha. Por extracción de cosecha, Soto (2002) indica que en una plantación de banano con un rendimiento de $30 \mathrm{t} / \mathrm{ha} / \mathrm{año}$ de fruta, es necesario para compensar las extracciones de N-P-K, suministrar $60 \mathrm{~kg}$ de $\mathrm{N}, 12,5 \mathrm{~kg}$ de $\mathrm{P}_{2} \mathrm{O}_{5}$ y $100 \mathrm{~kg} \mathrm{~K} \mathrm{O}_{2}$. En este mismo sentido, Twyford y Walmsley (1974) llegan a resultados similares e indican que para compensar una extracción de nutrimentos (N-P-K-Mg) contenidos en 1853 racimos de cv "Valery" por ha/año es necesario agregar $56,3 \mathrm{~kg}$ de $\mathrm{N}, 24,3 \mathrm{~kg}$ de $\mathrm{P}_{2} \mathrm{O}_{5}, 220,5 \mathrm{~kg}$ de $\mathrm{K}_{2} \mathrm{O}$ y $8,8 \mathrm{~kg}$ de $\mathrm{MgO}$. En cambio, Prével (1962) informó que por cada tonelada de fruta extraída, se debe agregar 2,0 kg de N, 0,5 kg de $\mathrm{P}_{2} \mathrm{O}_{5}$ y 6,0 kg de $\mathrm{K}_{2} \mathrm{O}$. Por otro lado, Tavares y Falquez (1997), indican que para una producción de 60 t/ha en un ciclo vegetativo del clon "Gran Enano", la extracción total de nutrimentos es de $101,97 \mathrm{~kg} \mathrm{~N}, 12,14 \mathrm{~kg} \mathrm{P}, 242,53 \mathrm{~kg} \mathrm{~K}$, $6,44 \mathrm{~kg} \mathrm{Ca}, 13,81 \mathrm{~kg} \mathrm{Mg}$ y $5,24 \mathrm{~kg} \mathrm{~S}$. Respecto a la extracción de elementos menores estos señalan que de Fe se extrae 0,36 kg, de Mn 0,13 kg, de Zn 0,10 kg, de B $0,09 \mathrm{~kg}$ y de $\mathrm{Cu} 0,04 \mathrm{~kg}$.

Parte importante de los nutrimentos extraídos por la planta provienen del proceso de descomposición natural del rastrojo (residuos de cosecha) del banano en campo. En sistemas de alta productividad de este cultivo, permanecen en los residuos 199, 23, 660, 126,76 y $50 \mathrm{~kg} / \mathrm{ha} /$ año en promedio de nitrógeno $(\mathrm{N})$, fósforo $(\mathrm{P})$, potasio $(\mathrm{K})$, calcio $(\mathrm{Ca})$, magnesio $(\mathrm{Mg})$ y azufre (S) respectivamente (Lahav y Turner 1983). Por otra parte, estudios realizados en fincas al este del río Reventazón, indican que por cada tonelada de fruta fresca se genera una tonelada de materia seca en rastrojo; a su vez, este contiene 7,05 kg de N; 5,19 de $\mathrm{kg}$ de Ca; 1,7 de $\mathrm{kg} \mathrm{Mg} ; 40,07 \mathrm{~kg}$ de $\mathrm{K} ; 1,47 \mathrm{~kg}$ de $\mathrm{P}$ y $0,75 \mathrm{~kg}$ de $\mathrm{S}$ (Vargas y Flores 1995).

Las pérdidas de nutrimentos por lixiviación en los suelos dedicados al cultivo de banano, localizados en las áreas al este y oeste del río Reventazón, en Costa Rica, podrían alcanzar valores altos, ya que estos suelos están sujetos a elevadas precipitaciones anuales (entre 3018 y $3719 \mathrm{~mm}$ ) y altas intensidades de lluvia en algunas épocas del año (López y Solís 1992), lo que favorece la percolación. Por otra parte, la aplicación de dosis altas de fertilizantes bajo las condiciones de suelo y clima prevalecientes en estas zonas, favorece el fenómeno de lixiviación (SSSA 1997). Por consiguiente, uno de los aspectos importantes a considerar en la aplicación de fertilizante al suelo en la zona Atlántica de Costa Rica, es el alto potencial de pérdidas de nutrimentos por lixiviación a través de la percolación del agua de lluvia, debido al origen aluvial volcánico y calcáreo de la mayoría de los suelos en esta área, ya que presentan características físicas de alta infiltración y conductividad hidráulica, lo que permite un rápido movimiento vertical (percolación) del agua en el perfil del suelo (Soto 1995).

Considerar el balance hídrico (BH) como una herramienta básica para estimar el volumen de agua percolada, es importante, ya que se relacionan los intercambios de agua entre la planta, el suelo y la atmósfera. Mediante el mismo, es posible establecer las ganancias y pérdidas de agua que se registra en un área dada, ya que permite hacer las estimaciones cuantitativas de la lixiviación de nutrimentos y pesticidas a través del agua de percolación (Jiménez 2004). Por ejemplo, Godefroy et al. (1975) en suelos bananeros de Costa de Marfil, estimó pérdidas de 210, 415 y 175 $\mathrm{kg} / \mathrm{ha} / \mathrm{año}$ de $\mathrm{N}, \mathrm{K}_{2} \mathrm{O}$ y $\mathrm{MgO}$ respectivamente. Por otra parte, Lahav y Turner (1983 y 1992) durante un periodo de ocho años de investigación en suelos dedicados al cultivo de banano de baja capacidad de intercambio catiónico $(5-10 \mathrm{cmol} / \mathrm{l})$ y alta precipitación (1400-2000 mm/año), logró estimar pérdidas por efecto de la lixiviación de alrededor de 165, 22, 376, 89 y $360 \mathrm{~kg}$ ha/año de N, P, K, Mg y Ca, respectivamente.

En banano se tienen datos preliminares sobre la pérdida de nutrimentos en diferentes tipos y condiciones de suelos y clima de Costa Rica, debido a la lixiviación. Durante 39 semanas las pérdidas de elementos en las aguas de drenaje subterráneo en un suelo bananero franco arcilloso de Siquirres, 
provincia de Limón, fueron obtenidas por González (1989), donde encontró valores de 39, 33, 842 y 354 $\mathrm{kg} / \mathrm{ha}$ de $\mathrm{N}, \mathrm{K}, \mathrm{Ca}$ y $\mathrm{Mg}$ respectivamente. Por otra parte, en Guápiles de Limón, en un suelo clasificado como Typic Hapludands, de textura liviana y con una alta capacidad de infiltración, se llegó a determinar pérdidas de alrededor de $111 ; 368 ; 123$ y $29 \mathrm{~kg} / \mathrm{ha} / \mathrm{año}$ de $\mathrm{K}, \mathrm{Ca}, \mathrm{Mg}$ y $\mathrm{NO}_{3}^{-2}$, respectivamente (Rosales et al. 1994). Por su parte Flores (1994) en ocho drenajes subterráneos (terciarios) encontró una alta correlación entre la precipitación y la pérdida de nutrimentos en suelos dedicados al cultivo del banano en la zona Atlántica de Costa Rica; según este autor, el catión que más se lixivió al este del río Reventazón fue el Ca, seguido por el $\mathrm{Mg}$ y luego el K, con 889; 350 y $34 \mathrm{~kg}$ ha/año, respectivamente. El objetivo de este trabajo fue determinar la pérdida por lixiviación de $\mathrm{Ca}, \mathrm{Mg}, \mathrm{K}$ y nitrógeno nítrico en suelos bananeros.

\section{MATERIALES Y MÉTODOS}

Ubicación y características climáticas del sitio experimental. Se seleccionaron lotes representativos de suelos utilizados en la explotación bananera al este del río Reventazón. Para ello se escogió la Finca Venecia, que se encuentra ubicada cerca del poblado de Venecia en Matina de Limón. Geográficamente, esta se localiza entre las coordenadas Lambert Norte 223 000-227 000 y 618 000-621 000 y el área experimental en las coordenadas 618755 y 224995 de las hojas cartográficas Matina 3546 III y Moín 3546 II, a una altitud que varía de 10 a $14 \mathrm{msnm}$. Ecológicamente, la zona en estudio pertenece al Bosque húmedo tropical transición a perhúmedo (bmh-P $\mathbf{\nabla})$ (Tosi 1969) y el tipo de clima según Herrera (1985) se define como húmedo muy caliente, con una estación seca corta ( $<35$ días intermitentes con déficit de agua) con una temperatura media anual de 25 a $27^{\circ} \mathrm{C}$ y una precipitación media anual de 3100 a $3500 \mathrm{~mm}$. Por su parte, los datos climáticos del área estudiada (promedio de diez años) procedentes del pluviómetro de la Finca Venecia y de la estación meteorológica de la Finca Bananita, indican que la precipitación total promedio anual es de 4201,8 mm, con promedios mensuales que oscilan de $88,8 \mathrm{~mm}$ para septiembre y hasta $542,5 \mathrm{~mm}$ para diciembre, distribuida con dos períodos de baja precipitación, uno durante los meses de marzo a abril y el otro de septiembre a octubre a lo largo del año. La evapotranspiración potencial promedio mensual es de $143,5 \mathrm{~mm}$, la temperatura media anuales es de $25,3^{\circ} \mathrm{C}$

Descripción del área experimental y análisis realizados. El área experimental se localizó en la tapa derecha del cable 40 entre las torres 8 y 17 de la Finca Venecia, la cual consta de tres parcelas (boquetes), cuyas medidas fueron de $27,7 \mathrm{~m}$ de ancho por 45,0 m de largo. Cada parcela se subdividió en tres bloques de 19,5 m de ancho por $12 \mathrm{~m}$ de largo para un total de nueve bloques, en donde se distribuyeron las nueve parcelas experimentales. Los métodos para las determinaciones analíticas de $\mathrm{pH}$ y concentración de $\mathrm{Ca}, \mathrm{Mg}, \mathrm{K}$ y N-nítrico en el agua de percolación fueron los utilizados en Costa Rica por el Laboratorio de Suelos del Ministerio y Agricultura y Ganadería (MAG) (Schweizer et al. 1980).

Tratamientos y diseño experimental. En cuanto a los tratamientos, estos consistieron en el uso de tres fuentes nitrogenadas: urea (U), nitrato de amonio (NA) y sulfato de amonio (SA) aplicados en cantidades equivalentes a $434 \mathrm{~kg} \mathrm{~N}$ ha/año y distribuidos de forma proporcional en un total de seis aplicaciones a razón de una aplicación por mes en forma diluida. Además, en todos los casos se aplicó una base general de $\mathrm{P}, \mathrm{K}, \mathrm{Mg}$ y $\mathrm{S}$ como mezcla física equivalente a: $108,0 \mathrm{~kg}$ de $\mathrm{P}_{2} \mathrm{O}_{5}$ en forma de fosfato diamónico (DAP); $680,4 \mathrm{~kg}$ de $\mathrm{K}_{2} \mathrm{O}$ como cloruro de potasio (KCL) y sulfato de potasio y magnesio $(\mathrm{K}-\mathrm{Mg})$ con $54,0 \mathrm{~kg}$ de $\mathrm{MgO}$ y $64,8 \mathrm{~kg}$ de $\mathrm{S}$ en forma de sulfato en la banda de abonamiento.

El diseño experimental utilizado fue de bloques completos al azar con tres tratamientos, que corresponden a tres fuentes nitrogenadas: urea (U), nitrato de amonio (NA) y sulfato de amonio (SA), con tres repeticiones. Por tratamiento y para cada una de las repeticiones se escogió dentro de cada parcela experimental una planta en estado fenológico Fm (emisión de la primera hoja verdadera) para instalar en la banda de abonamiento dos muestreadores de agua o lisímetros a diferentes profundidades, uno a $60 \mathrm{~cm}$ y el otro a $90 \mathrm{~cm}$. En total se instalaron dieciocho muestreadores de agua a razón de seis por tratamiento.

Muestreo de la solución del suelo. Los lisímetros constaron de un tubo de PVC de 2,5 cm de diámetro interno cuya longitud varió de 60 a $90 \mathrm{~cm}$, y estos en su extremo inferior presentan una copa porosa permeable a la solución del suelo y en el extremo 
superior un tapón de hule con un agujero, desde el cual se aplicó una presión de succión de 60 a 70 centibares, por medio de una bomba manual (Ramos y Kücke 1999). Los muestreadores de agua se instalaron en un agujero en el suelo según la profundidad deseada, el cual se realizó utilizando un barreno con un diámetro superior a estos. Las frecuencias de la extracción de los lixiviados fueron: cada quince días, durante los meses de octubre y noviembre del 2003; a partir de diciembre del 2003 a mayo del 2004, cada ocho días registrándose un total de 28 muestreos durante el periodo experimental.

Cálculo del volumen de agua de percolación mediante el método indirecto del balance hídrico del suelo (BHs). En este método, el valor de $D$ o el volumen de agua percolada, se calculó de forma diaria en función de los otros términos del balance de agua de la capa de suelo a $60 \mathrm{~cm}$ de profundidad. Según Soto (1995), es donde se acumula alrededor del $85 \%$ de las raíces del banano. Para tal fin se utilizó la metodología propuesta por el Centro Científico Tropical (Ewel et al. 1968) en donde:

D = Lluvia - Evapotranspiración del banano $\pm \mathbf{\Delta} H$

En donde, $\boldsymbol{\Delta} \mathrm{H}$ es el cambio del contenido de agua almacenada en el suelo. Por tal motivo, fue necesario calcular el valor de la capacidad de campo (CC) en mm (lámina) por horizontes o estratos del suelo del sitio experimental hasta la profundidad de $60 \mathrm{~cm}$. En esta ecuación, se supone que la percolación del agua es vertical y no hay escorrentía superficial, por lo plano $(<1 \%$ de pendiente) del terreno de la finca estudiada. La evapotranspiración potencial (ETP) se estimó en base a la fórmula propuesta por Ewel et al. 1968, donde:

$$
\mathrm{ETP}(\mathrm{mm}) \text { diaria }=\text { biotemperatura } * 58,93 / 365
$$

La biotemperatura (Ewel et al. 1968) se define como un promedio de las temperaturas en ${ }^{\circ} \mathrm{C}$ a las cuales tiene lugar crecimiento vegetativo, en relación con el periodo anual y se calcula a través de la fórmula empírica:

Biotemperatura $=\mathrm{T}^{\circ}$ media mensual - $(3 *$ grados de latitud $) / 100) *\left(\mathrm{~T}^{\circ} \text { media }-24\right)^{2}$
En el caso específico, la biotemperatura de la Finca Venecia se calculó diariamente de la siguiente manera:

Biotemperatura $=($ temperatura promedio diaria $)-[(3$ $\mathrm{x}(0+2,5 / 60) / 100) *$ temperatura promedio diaria-24] $]^{2}$

Biotemperatura $=($ temperatura promedio diaria $)-$ $[0,3012 * \text { temperatura promedio diaria- } 24]^{2}$

La evapotranspiración real (ETR) del banano se calculó en base al coeficiente del banano (Kc) de 1,2.

Cálculo de las pérdidas de nutrimentos por percolación. La lixiviación de los nutrimentos se midió al calcular el flujo gravitacional de la solución del agua del suelo (drenaje) por medio de método indirecto del balance hídrico del suelo (BHs) (Ewel et al. 1968) y la concentración media de los nutrimentos de la solución del agua del suelo en condiciones de campo según los datos obtenidos con los muestreadores de agua. Para tal fin, se dedujo la pérdida de agua por percolación profunda en el período entre muestreos en milímetros (mm) o litros $/ \mathrm{m}^{2}$ a partir del BHs de la zona más profunda, $60 \mathrm{~cm}$. En caso de existir pérdidas de agua por percolación profunda durante cada periodo de muestreo, estas se multiplican por la concentración de los nutrimentos correspondientes, obtenidos en los análisis de los lixiviados y de esta forma, se calculó las pérdidas de nutrimentos por lixiviación en kilogramos por hectárea (Jiménez 2004) con la fórmula:

$$
\mathrm{Ni}=\mathrm{Ppi} *[\mathrm{~N}] * 10000 \mathrm{~m}^{2} / \mathrm{ha} * \mathrm{~kg} / 1 \times 10^{6} \mathrm{mg}
$$

donde:

$\mathrm{Ni}$ : Masa de cualquier nutrimento por hectárea, por cada muestreo realizado y tratamiento $(\mathrm{kg} / \mathrm{ha})$.

Ppi: Pérdida de agua por percolación profunda en el periodo considerado $\left(\mathrm{mm} \mathrm{o} 1 / \mathrm{m}^{2}\right)$.

$[\mathrm{N}]$ : Concentración de cualquier nutrimento en el lixiviado recogido al final de cada periodo muestreado (mg/l).

Finalmente se sumaron los valores obtenidos de la fórmula anterior a lo largo del periodo de la investigación para cada tratamiento con el fin de calcular la masa de cualquier nutrimento considerado por hectárea y tratamiento lixiviado a lo largo del periodo de estudio, mediante la ecuación: 


$$
\mathrm{N}_{\mathrm{T}}=\Sigma \mathrm{Ni}
$$

Donde,

$\mathrm{N}_{\mathrm{T}}$ Masa de cualquier nutrimento considerado por hectárea y tratamiento $(\mathrm{kg} / \mathrm{ha})$

$\mathrm{Ni}$ : Masa de cualquier nutrimento considerado por hectárea, periodo y tratamiento $(\mathrm{kg} / \mathrm{ha})$

Análisis de la información. Para el análisis estadístico de los datos se usó el programa INFOSTAT (Balzarini et al. 2008). Cuando se determinaron diferencias significativas, estas se calcularon con una probabilidad de $\mathrm{p}<0,05$.

\section{RESULTADOS Y DISCUSIÓN}

Variación de las características químicas y las pérdidas por lixiviación de nutrimentos para los tratamientos de urea, nitrato de amonio y sulfato de amonio

pH. Los promedios generales fueron de 6,59; 6,53 y 6,62 para los tratamientos de U, NA y SA respectivamente (Cuadro 1), independientemente de las fechas de muestreo no resultaron ser diferentes estadísticamente $(p \geq 0,05)$. Situación que podría ser explicada por la capacidad tampón que presentaron estos suelos para amortiguar los efectos de cambio de $\mathrm{pH}$ por diversos tipos de sustancias presentes en el medio (Fassbender y Bornemisza 1994, Tisdale y Nelson 1970). En el sitio bajo estudio, esta condición tampón (buffer) se debe al origen aluvial calcáreo de los suelos, que presentan altos contenidos de bases $(28,16$ a $32,8 \mathrm{cmol} / \mathrm{l}$ ) principalmente de $\mathrm{Ca} \mathrm{y} \mathrm{Mg}$, que ocupan el mayor porcentaje de la suma de bases presentes en el complejo de cambio (Acón 2002). Asimismo, debe considerarse la presencia de ácidos húmicos estables (procedente de la mineralización de la materia orgánica) que actúan como intercambiador de cationes reaccionado con los iones $\mathrm{H}+$ (Crovetto 2002).

Se observa, que en los muestreos que presentan alta precipitación estacional la tendencia general en los tratamientos, es bajar levemente los valores de $\mathrm{pH}$, debido al mayor metabolismo en las plantas de banano asociados con altas producciones. Lo anterior es consecuencia de que para balancear las cargas dentro de la planta hay un intercambio iónico durante la absorción radical (exudaciones de la raíz), debido a que cuando una base o catión es absorbida por las raíces la planta expulsa a la solución de suelo una cantidad equivalente de $\mathrm{H}^{+}$(Fassbender y Bornemisza 1994, Bertsch 1998) y durante los meses de mayor pluviosidad estacional, por la baja permeabilidad de estos suelos ocurre la saturación de los mismos, dando como resultado que durante el proceso de la respiración del banano se produce $\mathrm{CO}_{2}$ por las raíces y parte del mismo no se intercambia al medio ambiente reaccione con los $\mathrm{H}^{+}$ y $\mathrm{OH}^{-}$constituyentes del agua, formando iones $\mathrm{H}^{+}$y $\mathrm{HCO}_{3}^{-}$, lo que disminuye el pH del suelo (Fassbender y Bornemisza 1994).

Calcio. Los resultados obtenidos en relación con la dinámica de la concentración de calcio $(\mathrm{Ca})$ en los lixiviados durante las diferentes épocas de muestreos (Cuadro 1), indica que independientemente de la época, los tratamientos mostraron diferencias significativas $(\mathrm{p} \leq 0,05)$ de la $\mathrm{U}$ con un valor de $1,98 \mathrm{mg} / \mathrm{l}$ respecto al NA y SA, con valores de 2,72 y 2,91 mg/l respectivamente; no encontrándose diferencias entre el NA y SA.

Los datos evidencian que el tratamiento con $\mathrm{U}$ presenta menos $\mathrm{Ca}$ en el lixiviado, que con respecto a los tratamientos de NA y $\mathrm{SA}$, lo que podría relacionarse, a las fuentes de iones acompañantes $\mathrm{NO}_{3}^{-} \mathrm{y}$ $\mathrm{SO}_{4}^{-2}$ que permiten la movilidad de los iones básicos y por el otro lado, el efecto que tiene el $\mathrm{NH}_{4}^{+}$como ión desplazante de los cationes $\mathrm{Ca}, \mathrm{Mg}$ y $\mathrm{K}$ de la micela coloidal (Fassbender y Bornemisza 1994, López y Espinosa 1995). La variación de la concentración mensual del Ca entre los tratamientos de U y NA (Cuadro 1) muestra una tendencia de mayor concentración en los lixiviados en el tratamiento de NA, en los meses de mayor precipitación estacional (diciembre: 1094,8 mm y marzo: $518,0 \mathrm{~mm}$ ) y al mes siguiente, lo que podría ser relacionado a condiciones de suelo más húmedo y que genera mayor cantidad de nitratos $\left(\mathrm{NO}_{3}^{-}\right)$producidos por el proceso nitrificación del amonio $\left(\mathrm{NH}_{4}^{+}\right)$ (González et al. 1985).

Ligado a esta situación, el $\mathrm{NO}_{3}{ }^{-}$aplicado al suelo como fertilizante, actuaron como acarreador o ión acompañantes del $\mathrm{Ca}$, lo que fomenta de esta forma una mayor movilidad y concentración del mismo en los lixiviados (Fassbender y Bornemisza 1994, López y Espinosa 1995). Respecto al tratamiento con U, y la baja presencia de $\mathrm{Ca}$ en los lixiviados, esto podría relacionarse a las condiciones de alta cantidad de agua 
Cuadro 1. Dinámica del valor de $\mathrm{pH}$ y la concentración de calcio, magnesio y potasio (mg/l) durante los muestreos de lixiviados, según la fuente de nitrógeno empleada. Matina, Limón, Costa Rica. 2003-2004.

\begin{tabular}{|c|c|c|c|c|c|c|c|c|c|c|c|}
\hline \multirow[t]{3}{*}{ Muestreo } & \multirow{3}{*}{$\begin{array}{c}\text { Lluvia } \\
(\mathbf{m m})\end{array}$} & \multirow{3}{*}{$\begin{array}{l}\text { Percolación } \\
\quad(\mathbf{m m})\end{array}$} & \multicolumn{9}{|c|}{ Variables químicas del suelo* } \\
\hline & & & \multicolumn{3}{|c|}{ pH } & \multicolumn{3}{|c|}{ Calcio (mg/l) } & \multicolumn{3}{|c|}{ Magnesio (mg/l) } \\
\hline & & & $\mathbf{U}$ & NA & SA & $\mathbf{U}$ & NA & SA & $\mathbf{U}$ & NA & SA \\
\hline I & 307,8 & 192,3 & $6,55 \mathrm{a}$ & $6,80 \mathrm{a}$ & $6,81 \mathrm{a}$ & $2,58 \mathrm{a}$ & $2,54 \mathrm{a}$ & $3,38 \mathrm{~b}$ & $0,97 \mathrm{a}$ & $1,00 \mathrm{ab}$ & $1,02 \mathrm{~b}$ \\
\hline II & 222,3 & 152,8 & $6,65 \mathrm{a}$ & $6,95 \mathrm{a}$ & $6,89 \mathrm{a}$ & $2,10 \mathrm{a}$ & $3,29 b$ & $3,12 b$ & $0,96 \mathrm{a}$ & $0,99 a$ & $0,98 \mathrm{a}$ \\
\hline III & 1027,4 & 921,5 & $6,35 \mathrm{a}$ & $6,29 \mathrm{a}$ & $6,61 \mathrm{a}$ & $2,12 \mathrm{a}$ & $3,14 b$ & $3,31 b$ & $0,99 \mathrm{a}$ & $0,95 \mathrm{a}$ & $1,07 \mathrm{~b}$ \\
\hline IV & 139,3 & 80,1 & $6,46 a$ & $6,57 \mathrm{a}$ & $6,84 \mathrm{a}$ & $1,13 \mathrm{a}$ & $2,53 b$ & $3,16 b$ & $0,80 \mathrm{a}$ & $0,95 \mathrm{ab}$ & $1,06 \mathrm{~b}$ \\
\hline V & 286,1 & 217,2 & $7,08 \mathrm{a}$ & $6,62 \mathrm{a}$ & $7,07 \mathrm{a}$ & $2,15 \mathrm{a}$ & $2,74 \mathrm{a}$ & $2,57 \mathrm{a}$ & $1,02 \mathrm{a}$ & $1,14 \mathrm{~b}$ & $1,00 \mathrm{a}$ \\
\hline VI & 560,8 & 441,7 & $6,57 \mathrm{a}$ & $6,49 a$ & $6,78 \mathrm{a}$ & $2,10 \mathrm{a}$ & $2,69 b$ & $3,11 \mathrm{~b}$ & $0,98 \mathrm{a}$ & $1,00 \mathrm{ab}$ & $1,05 \mathrm{~b}$ \\
\hline VII & 248,5 & 179,7 & $7,00 \mathrm{c}$ & - & $5,97 \mathrm{~b}$ & $1,37 \mathrm{~b}$ & - & $2,40 \mathrm{a}$ & $0,97 \mathrm{~b}$ & - & $0,83 b$ \\
\hline VIII & 947,3 & 831,1 & $6,04 \mathrm{a}$ & $5,99 \mathrm{a}$ & $6,02 \mathrm{a}$ & $2,28 \mathrm{a}$ & $2,09 \mathrm{a}$ & $2,22 \mathrm{a}$ & $0,78 \mathrm{a}$ & $0,79 \mathrm{a}$ & $0,78 \mathrm{a}$ \\
\hline Promedio & 3738,3 & 3016,4 & $6,59 \mathrm{a}$ & $6,53 a$ & $6,62 a$ & $1,98 \mathrm{a}$ & $2,72 b$ & 2,91b & $\mathbf{0 , 9 3 a}$ & $0,97 \mathbf{a}$ & $0,97 \mathbf{a}$ \\
\hline \multirow[t]{2}{*}{ Muestreo } & & & \multicolumn{3}{|c|}{ Potasio (mg/l) } & \multicolumn{3}{|c|}{ N-nítrico (mg/l) } & & & \\
\hline & & & $\mathbf{U}$ & NA & SA & $\mathbf{U}$ & NA & SA & & & \\
\hline I & & & $0,34 \mathrm{a}$ & $0,16 \mathrm{a}$ & $0,28 \mathrm{a}$ & $13,69 \mathrm{a}$ & $5,43 \mathrm{~b}$ & $12,37 \mathrm{a}$ & & & \\
\hline II & & & $0,09 \mathrm{a}$ & $0,26 \mathrm{a}$ & $0,12 \mathrm{a}$ & $15,75 \mathrm{a}$ & $6,95 b$ & $13,05 \mathrm{a}$ & & & \\
\hline III & & & $0,09 \mathrm{a}$ & $0,08 \mathrm{a}$ & $0,12 \mathrm{a}$ & $12,74 \mathrm{a}$ & $6,29 b$ & $13,97 \mathrm{a}$ & & & \\
\hline IV & & & $0,09 \mathrm{ab}$ & $0,08 \mathrm{a}$ & $0,13 b$ & $11,12 \mathrm{a}$ & $6,57 b$ & $11,57 \mathrm{a}$ & & & \\
\hline V & & & $0,09 \mathrm{a}$ & $0,09 \mathrm{a}$ & $0,14 \mathrm{a}$ & $11,29 \mathrm{a}$ & $6,62 b$ & $10,85 \mathrm{a}$ & & & \\
\hline VI & & & $0,08 \mathrm{a}$ & $0,07 \mathrm{a}$ & $0,13 \mathrm{a}$ & $11,02 \mathrm{a}$ & $6,49 b$ & $11,12 \mathrm{a}$ & & & \\
\hline VII & & & $0,09 \mathrm{~b}$ & - & $0,13 b$ & $16,95 b$ & - & $8,53 b$ & & & \\
\hline VIII & & & $0,14 \mathrm{a}$ & $0,11 \mathrm{a}$ & $0,12 \mathrm{a}$ & $11,67 \mathrm{a}$ & $5,99 \mathrm{~b}$ & $10,53 \mathrm{a}$ & & & \\
\hline Promedio & & & $0,13 a$ & $0,12 \mathrm{a}$ & $0,15 a$ & $13,03 a$ & $6,33 b$ & $11,50 a$ & & & \\
\hline
\end{tabular}

* $\mathrm{U}=$ urea, $\mathrm{NA}=$ nitrato de amonio, $\mathrm{SA}=$ sulfato de amonio.

Letras diferentes en una misma columna difieren significativamente $(\mathrm{p}<0,05)$.

en el suelo y por las bajas temperaturas. Los procesos de hidrólisis de la U podrían ser más lentos, por consiguiente, también se reduce la producción del $\mathrm{NH}_{4}^{+}$ y de $\mathrm{NO}_{3}^{-}$; y por otra parte, durante la hidrólisis de la $\mathrm{U}$, se podrían perder cantidades apreciables de $\mathrm{NH}_{3}$ por volatilización cuando se está evaporando agua de la superficie y el suelo carece de coberturas como se manejan las plantaciones de banano.

Entre los tratamientos de U y SA, se observó una mayor tendenciaen el SAde incrementar la concentración en los lixiviados respecto al de U (Cuadro 1), lo que se relaciona a la capacidad del ion sulfato $\left(\mathrm{SO}_{4}^{2-}\right)$ de ser un anión acompañante, que permite la movilidad del
Ca y otros cationes como el potasio $(\mathrm{K}+)$ y el magnesio $\left(\mathrm{Mg}^{2+}\right)$ (López y Espinosa 1995). Además, Fassbender y Bornemisza (1994), indican que en suelos ubicados en Puerto Rico, el 90\% de los cationes lavados se perdió por aplicaciones de fertilizantes sulfatados, debido a la capacidad del sulfato como anión acompañante y la reacción ácida que presente en el suelo. En relación con los tratamientos de NA y SA, presentan el mismo efecto, debido a que movilizan las mismas concentraciones de $\mathrm{Ca}$ en los lixiviados obtenidos en forma mensual $(\mathrm{p} \geq 0,05)$ (Cuadro 1).

Magnesio. En cuanto a los promedios de las concentraciones de magnesio $(\mathrm{Mg})$ en los lixiviados 
(Cuadro 1), se observó que independientemente de las fechas de muestreo entre los tratamientos no existieron diferencias significativas ( $\mathrm{p} \geq 0,05)$, cuyos valores fluctuaron entre $0,93 \mathrm{mg} / \mathrm{l}$ para la $\mathrm{U}$ y de $0,97 \mathrm{mg} / \mathrm{l}$ tanto para SA y NA.

Potasio. En cuanto a la dinámica de las concentraciones de potasio (K) en los lixiviados, se observó un comportamiento similar al obtenido para $\mathrm{Mg}$, es decir no hay diferencias significativas según los promedios generales (Cuadro 1). Las concentraciones de $\mathrm{K}$ en los lixiviados en los tres tratamientos fueron parecidas durante todo el proceso; resultado similar al que informan González et al. (1985), en suelos volcánicos en donde el K presentó baja movilidad. En cuanto a las concentraciones de $\mathrm{K}$ en los lixiviados respecto a los meses, el análisis estadístico muestra que entre los distintos tratamientos, no hubo diferencias significativas $(\mathrm{p} \geq 0,05)$. Estos resultados indican, una baja movilidad del $\mathrm{K}$ en estos suelos, lo que podría relacionarse con que el $\mathrm{K}$, es retenido de forma electrostática (Tisdale y Nelson 1970), por presentar en los coloides una alta capacidad de intercambio catiónico $(48,15$ a 53,85 cmol/l) (López y Espinosa 1995) también, el K está sometido a procesos de fijación entre los espacios interlaminares de las arcillas 2:1 predominante en estos suelos (Arias 2009). Asimismo, debido a que la planta de banano es altamente extractora de este nutrimento, es posible que gran parte del fertilizante adicionado fuera absorbido y exportado del sistema a través de la producción de fruta (Flores 1994).

Nitrógeno nítrico. Sólo se detectaron diferencias significativas $(\mathrm{p} \leq 0,05)$ entre el NA respecto a la $\mathrm{U}$ y al SA, tanto en forma general como entre fechas de muestreo, en este caso, las menores concentraciones de $\mathrm{N}-\mathrm{NO}_{3}$ en los lixiviados del tratamiento de NA respecto a los tratamientos de $\mathrm{U}$ y $\mathrm{SA}$, lo cual tiene relación con las diferencias en la composición de las fuentes utilizadas, ya que el NA contiene $50 \%$ de nitratos y las otras fuentes no, así como la existencia de condiciones que favorecen el proceso de denitrificación biológica (Tisdale et al. 1993).

Entre las condiciones que favorecen este proceso, Fassbender y Bornemisza (1994) indican la presencia de alta humedad en el suelo, junto con otros factores como la concentracion de nitratos, temperutura y condiciones de redox del suelo. Por último, se considera la absorción de nitrato a nivel radicular por el cultivo, ya que es una forma accesible para la planta, por su movilidad en la solución del suelo por medio de difusión hacia las raíces (Ramírez 2001, Bertsch 1998), por lo tanto, estas van a disponer y absorber de más $\mathrm{NO}_{3}^{-}$que el asequible sólo por la interceptación radical.

\section{LITERATURA CITADA}

Acón, J. 2002. Estudio detallado de suelos y clasificación de las tierras según su aptitud para el cultivo de banano de la finca perteneciente a la Compañía Hacienda Río Palacio S.A., situada en Venecia y Saborío, Distrito Carrandí, Cantón Matina, Provincia de Limón. 84 p.

Arias, F. 2009 Caracterización química y mineralógica de suelos cultivos en banano (Musa AAA) en la zona Caribe de Costa Rica. Tesis Mag. Sc. San José, Costa Rica, Sistema de Estudios de Posgrado, Universidad de Costa Rica. 135 p.

Balzarini, MG; González, L; Tablada, M; Casanoves, F; Di Renzo, JA; Robledo, CW. 2008. Manual del usuario. InfoStat, versión 2008. Córdoba, Argentina, Editorial Brujas. 336 p.

Bertsch, F. 1998. La fertilidad de los suelos y su manejo. San José, Costa Rica, Asociación Costarricense de la Ciencia del Suelo (ACCS). 57 p.

Crovetto, C. 2002. Cero labranza. Los rastrojos, la nutrición del suelo y su relación con la fertilidad de las plantas. Talcahuano, Chile, Trama Impresoso S.A. 225 p.

Ewel, J; Madriz,A; Tosi, J. 1968. Zonas de vida de Venezuela: Memoria explicativa sobre el mapa ecológico. Fondo Nacional de Investigaciones Agropecuarias, Ministerio de Agricultura y Cría, Caracas, República de Venezuela. 264 p.

Fassbender, H; Bornemisza, E. 1994. Química de suelos con énfasis en suelos de América Latina. San José, Costa Rica, IICA. 420 p.

Flores, C. 1994. Pérdida de cationes y aniones en suelos bananeros de la Zona Atlántica de Costa Rica. In Resúmenes de XI ACORBAT. San José, Costa Rica. $18 \mathrm{p}$.

Godefroy, J; Roose, E; Muller, M. 1975. Estimation des parts par les eaux de ruisellement et de drainage des elements fertilisants dans un sol de bananeraie de sud de la Cote d'Ivore. Fruits 30(4):223-235.

González, MA; Pacheco, R; Briceño, JA. 1985. Efecto de la úrea y del nitrato de amonio sobre la movilidad de nitrato, potasio, calcio y magnesio en un Dystrandept 
de Costa Rica. Agronomía Costarricense 9(2):241251.

González, P. 1989. Determinación de las pérdidas de aniones y cationes en el agua de drenaje subterráneo en un suelo bananero. Tesis Lic. San José, Costa Rica, Facultad de Agronomía, Universidad de Costa Rica. 138 p.

Herrera, W. 1985. Clima de Costa Rica. San José, Costa Rica, Universidad Estatal a Distancia (UNED). 118 p.

Jiménez, F. 2004. Medición de los componentes del balance hídrico en sistemas agroforestales. Curso metodología de investigación en sistemas agroforestales. Turrialba, Costa Rica, CATIE. 25 p.

Lahav, E; Turner, DW. 1983. Fertilizing for high yield banana. IPI-Bulletin 7. Berne, Switzerland, International Potash Institute. 38 p.

Lahav, E; Turner, DW. 1992. Nutrición del banano. 2 ed. rev. Boletín No 7. Quito, Ecuador, Instituto de la Potasa y el Fósforo. 71 p.

López, A. 1991. Fertilización del cultivo de banano con diferentes dosis de nitrógeno, fósforo y potasio. In Informe Anual, Corporación Bananera Nacional S.A. San José, Costa Rica. p. 35-36.

López, A; Solís, P. 1992. Contenidos e interacciones de los nutrientes en tres zonas bananeras de Costa Rica. CORBANA (C.R.) 15(36):25-32.

López, A; Espinosa, J. 1995. Manual de nutrición y fertilización del banano. Quito, Ecuador, Instituto de la Potasa y el Fósforo. 82 p.

Prevel, P. 1962. Les élements mineraux dans le bananier et dans son régime. Fruits 17(3):123-128.

Ramírez, ZC. 2001. Nutrición nitrogenada de las plantas. In Villalobos, E. ed. Fisiología de la producción de los cultivos tropicales. San José, C.R, Editorial de la Universidad de Costa Rica. p. 198-226.

Ramos, C; Kücke, M. 1999. Revisión crítica de los métodos de medida de la lixiviación de nitrato en suelos agrícolas. Estudios de la zona no saturada del suelo.
Eds. R. Muñoz-Carpena, A. Ritter, C. Tascón. Tenerife, España, ICIA. p. 25-32.

Rosales, A; Maebe, P; Sevenhuysen, R. 1994. Determinación de las pérdidas de nutrimentos y nematicidas en las aguas de drenaje de un suelo bananero. Agronomía Costarricense 18(1):93-98.

Schweizer, S; Coward, H; Vasquez, A. 1980. Metodología para análisis de suelos, plantas y agua. San José, Costa Rica, Ministerio de Agricultura y Ganadería. 25 p.

Soto, M. 1995. Bananos: cultivo y comercialización. 2 ed. San José, Costa Rica, LIL. 649 p.

SSSA (Soil Science Society of America). 1997. Glossary of soil science terms. Madison, Wi, Soil Science Society of America Inc. 37 p.

Tavares, G; Falquez, C. 1997. Contenido nutricional y absorción de nutrientes en plantas de banano (Musa AAA), subgrupo Cavendish, clon "Gran Enano", en diferentes etapas fenológicas de desarrollo. Trabajo de Graduación. Guácimo, Costa Rica, EARTH. 77 p.

Tisdale, S; Nelson, C. 1970. Fertilidad de los suelos y fertilizantes. Barcelona, España, Montaner y Simon, S.A. 760 p.

Tisdale, S; Nelson, W; Beaton, J; Havlin, J. 1993. Soil fertility and fertilizers. 5 ed. New York, United States of America, Macmillan Publishing Company. 632 p.

Tosi, JA. 1969. Mapa ecológico de Costa Rica. Según la clasificación de zonas de vida del mundo de Holdridge. Centro Científico Tropical, San José, Costa Rica. Escala 1:750.000.

Twyford, IT; Walmsley, D. 1974. The mineral composition of the Robusta banana plant. I. Methods and plant growth studies. Plants and Soil 39:227-243.

Vargas, R; Flores, CL. 1995. Retribución nutricional de los residuos orgánicos de hojas, venas de hojas, pseudotallos y pinzote de banano (Musa AAA) en fincas de diferentes edades de cultivo. CORBANA (C.R.) 20(44):33-47. 